\title{
O olhar do visitante: o caso do museu Espaço Memorial Carlos Chagas Filho
}

\section{The visitor's look: the Memorial Centre Carlos Chagas Filho case study}

\section{Gabriella da Silva Mendes}

Programa de Pós-Graduação do Instituto NUTES de Educação, Ciências e Saúde (PPGECS-NUTES), Universidade Federal do Rio de Janeiro (UFRJ).

gabiufrj1@gmail.com

orcid.org/0000-0002-1580-4859

\section{Thaís Patrícia Mancilio da Silva}

Programa de Pós-Graduação em História das Ciências e da Saúde (PPGHCS),

Fundação Oswaldo Cruz (Fiocruz)

thais.mancilio@gmail.com

orcid.org/0000-0002-8661-0226

\section{Pedro Henrique Bonini da Silva}

Programa de Pós-Graduação do Instituto NUTES de Educação, Ciências e Saúde (PPGECS-NUTES), Universidade Federal do Rio de Janeiro (UFRJ)

pedrohenriquebonini@gmail.com

orcid.org/0000-0003-3434-922X

\section{Humberto Martins de Souza}

Programa de Pós-Graduação do Instituto NUTES de Educação, Ciências e Saúde (PPGECS-NUTES), Universidade Federal do Rio de Janeiro (UFRJ)

hszmartins@gmail.com

orcid.org/0000-0001-5973-5085

\section{Vinicius Valentino Maria}

Instituto de Biofísica Carlos Chagas Filho (IBCCF), Universidade Federal do Rio de Janeiro (UFRJ)

vi.valent.vm@gmail.com

orcid.org/0000-0003-0303-5236

\section{Érika Negreiros}

Instituto de Biofísica Carlos Chagas Filho (IBCCF), Universidade Federal do Rio de Janeiro (UFRJ)

erikanegres@biof.ufrj.br

orcid.org/0000-0001-7325-1538 
Resumo. O Espaço Memorial Carlos Chagas Filho (EMCCF) é um museu de História da Ciência dedicado à preservação e à publicização da história do Instituto de Biofísica Carlos Chagas Filho (IBCCF) e da biografia de seu fundador, o renomado cientista brasileiro Carlos Chagas Filho. Espaços desse tipo são fundamentais para a manutenção da memória e para a extensão universitária. Por essa razão é muito importante que se criem parâmetros avaliativos visando perceber o impacto sobre seus diferentes públicos, compreender seus interesses e expectativas, produzir conhecimento e se remodelar de acordo com as demandas percebidas. Nesse sentido, o presente trabalho busca apresentar os resultados de um processo de avaliação realizado com visitantes do EMCCF ao longo de um ano. Esses dados foram obtidos a partir de questionários com perguntas abertas e fechadas aplicados a estudantes de escolas públicas e privadas, buscando apreender aspectos socioculturais dos visitantes e suas percepções sobre as experiências vivenciadas em sua visita. Para muitos, a ida ao EMCCF foi a primeira experiência de visita a um museu, enfatizando o papel da escola no acesso a espaços do gênero. Dentre os elementos marcantes na experiência da visita, se destacaram os momentos interativos, indicando a importância desse tipo de abordagem para uma experiência mais completa.

Palavras-chave: Museu. História da ciência. Divulgação científica.

\begin{abstract}
The Memorial Centre Carlos Chagas Filho (EMCCF) is a Science Museum dedicated to the preservation and promulgation of the history of the Institute of Biophysics Carlos Chagas Filho (IBCCF) and its founder, the renowned scientist Carlos Chagas Filho. Spaces such as this one are fundamental to the conservation of the institute's memory and to university extension programs. For that reason, it is very important to create assessment frameworks aiming to examine its impacts on its different groups of visitors, understand their interests and expectations, produce knowledge and remodel itself according to the demands observed. In that sense, this paper seeks to present the results of a process of evaluation carried out with EMCCF visitors during the course of one year. This data was obtained via surveys with open-ended and close-ended questions conducted with private and state school students, with the goal of learning about the visitors' socio-cultural backgrounds and their impressions of the experiences of their visit. For many, the trip to EMCCF was their first museum trip, which emphasizes the role of schools in providing access to places such as this one. Amongst the highlights of the experience, interactive moments stand out, indicating the importance of this type of approach to a more fully realised experience.
\end{abstract}

Keywords: Museum. Science history. Science dissemination.

Recebido: 01/10/2017. Aceito: 27/10/2017. Publicado: 06/11/2017 


\section{Introdução e Contextualização}

O Espaço Memorial Carlos Chagas Filho (EMCCF) é um museu de história da ciência localizado no Instituto de Biofísica Carlos Chagas Filho (IBCCF) da Universidade Federal do Rio de Janeiro (UFRJ). Esse espaço possui em suas dependências o escritório musealizado do renomado cientista Carlos Chagas Filho, além de exposições sobre temas relacionados às ciências naturais e uma reserva técnica com uma volumosa coleção de equipamentos científicos, livros e documentos. Foi fundado no ano 2000 com o objetivo principal de pesquisar, preservar e divulgar a memória do IBCCF, de seu fundador e dos diversos cientistas que a ele deram importantes contribuições. $\mathrm{O}$ EMCCF possui um perfil multifacetado, dada a variedade de atividades nele realizadas. É considerado um museu de história da ciência porque se preocupa com a preservação e a publicização de seu acervo histórico, porém é também um museu de ciências, pois realiza atividades de experimentação com temas relacionados às pesquisas desenvolvidas no IBCCF.

Em função da diversidade de estímulos oferecidos por suas exposições temáticas, museus de ciências podem ser considerados contextos privilegiados para a construção de diálogos compartilhados entre variados grupos (BONATTO et al, 2007). Por muito tempo esses espaços foram compreendidos como responsáveis por apresentar de maneira unilateral o conhecimento científico ao público leigo, a quem cabia unicamente contemplar as conquistas da ciência. Atualmente são considerados espaços que pensam em formas de aumentar a interatividade e que começam a entender a necessidade de engajar o público, torná-lo protagonista do funcionamento do museu, instigá-lo a participar de debates, catalisar formas de apropriação concreta e crítica da ciência e tecnologia (CASTELFRANCHI, 2016). E é exatamente esse tipo de experiência que o EMCCF procura providenciar ao seu público, tendo suas práticas orientadas pelos princípios da divulgação científica e da extensão, cujo exercício é mais do que a mera transferência de conhecimento, consistindo na busca constante por um maior diálogo entre universidade e sociedade.

Diante de tal perspectiva foi desenvolvido o projeto "Descobrindo a Biofísica", iniciativa que promove a visita de escolas da educação básica ao EMCCF. Por intermédio desse projeto há semanalmente a disponibilização de um ônibus para que estudantes da rede estadual e das redes municipais de ensino do Rio de Janeiro possam visitá-lo. Durante a visita, essas turmas participam de mediações nas exposições do EMCCF e de oficinas realizadas nos laboratórios do IBCCF, as quais buscam apresentar parte da rotina de pesquisadores da UFRJ. A presente pesquisa é uma parcela dos esforços realizados com o intuito de compreender a opinião desses estudantes sobre as experiências vivenciadas ao longo dessas visitas e, em especial, à exposição "A História da Ciência do Brasil: o capítulo do Instituto de Biofísica Carlos Chagas Filho". Este tipo de pesquisa é capaz de promover uma série de importantes reflexões sobre os limites e as potencialidades da relação entre espaços de educação formal e de educação não formal. 


\section{A Mediação no EMCCF}

É possível indicar o ano de 2015 como um marco importante para as reflexões que serão apresentadas ao longo desse trabalho. Naquele ano o IBCCF completou 70 anos de existência e, com o intuito de homenagear sua trajetória, foi desenvolvida a exposição interativa "A História da Ciência do Brasil: o capítulo do Instituto de Biofísica Carlos Chagas Filho", inaugurada no mês de dezembro daquele mesmo ano. O tema central da exposição foi baseado no livro "Construtores do Instituto de Biofísica Carlos Chagas Filho", lançado em 2013. Essa publicação consistia em uma homenagem póstuma aos primeiros pesquisadores do IBCCF que, juntamente com Carlos Chagas Filho, estabeleceram e consolidaram as pesquisas científicas desenvolvidas no instituto. $\mathrm{O}$ grande objetivo deste projeto foi preservar a memória do IBCCF e de seus primeiros pesquisadores, promovendo a popularização de uma parte da história da ciência brasileira.

Desenvolver uma exposição com uma grande densidade de conceitos e informações para um público composto majoritariamente por alunos da educação básica apresentouse como um grande desafio. O risco seria estabelecer uma exposição muito distante da realidade dos visitantes, com muita informação histórica e pouca interatividade, o que não teria um impacto positivo e não despertaria interesse. Por essa razão a exposição foi pensada com base nas áreas da ciência e não simplesmente na biografia de cada pesquisador isoladamente. Sua disposição foi composta por módulos temáticos que representam áreas de pesquisas científicas desenvolvidas no IBCCF (Bioquímica, Biologia Molecular, Biologia Celular, Microscopia, Neurobiologia e Radiobiologia), sendo dispostos em ordem e divididos por cores. Cada módulo conta a história dos cientistas de forma lúdica e com atividades interativas, mesclando conceitos científicos com elementos de experimentação e arte, de forma a promover uma maior interação entre os visitantes e os temas abordados.

A mediação humana foi um elemento considerado muito importante para atingir os objetivos predeterminados no desenvolvimento desta exposição. A presença de um elo que permitisse a aproximação dos discentes com os elementos que a constituiu demonstrou-se muitas vezes indispensável. Dessa maneira, todos os possíveis itinerários de mediação foram pensados com muita atenção e considerando a multiplicidade de públicos e de condições nas quais as visitas podiam se desenrolar. Todos os mediadores atuantes no EMCCF participaram do processo de construção desses roteiros, o que serviu para enriquecê-los significativamente, pois se tratava de um grupo bastante heterogêneo e multidisciplinar. Ressalta-se que esses roteiros serviram para guiar, não engessar as mediações, oferecendo assim um leque variado de possibilidades. Em todos os módulos a abordagem utilizada na mediação buscava suscitar questionamentos sobre os temas de ciências. Respostas prontas foram evitadas ao máximo, buscando assim desenvolver junto ao público um diálogo que propiciasse uma construção coletiva de conhecimentos significativos, algo que se demonstrou bastante adequado.

A partir da inauguração da exposição interativa, as visitas ao EMCCF cresceram exponencialmente. Desde sua inauguração, no final de 2015, até o término de 2019 a 
exposição recebeu cerca de 6000 visitantes, número $100 \%$ maior do que o registrado nos oito anos anteriores. Dessa maneira, é possível perceber que esse projeto vem contribuindo enormemente para consolidar o papel do EMCCF como um museu de história da ciência e divulgação científica dentro do IBCCF, onde pesquisa e extensão ocorrem de maneira interligada.

\section{Resultados}

Com o intuito de verificar a percepção do público escolar recebido desde a inauguração da exposição, a equipe do setor educativo do EMCCF buscou estratégias que visaram coletar informações sobre o perfil socioeconômico e cultural dos discentes, bem como suas percepções sobre a experiência vivida durante a visitação. Buscou-se então analisar as visitas realizadas no decorrer do ano de 2016, primeiro ano da exposição. A avaliação inicial foi desenvolvida a partir de 178 questionários, no qual 90\% eram referentes a estudantes de escolas públicas e $10 \%$ de estudantes de escolas particulares. O questionário foi composto por perguntas abertas e fechadas, sendo aplicado ao final da visita. Este tipo de pesquisa, além de revelar a percepção do público, também pôde dimensionar resultados da exposição e avaliar sua pertinência e eficácia. A análise dos dados permitiu observarmos que a maioria dos alunos investigados não frequenta museus e que essa foi a sua primeira experiência em um espaço de educação não formal (Figura 1).

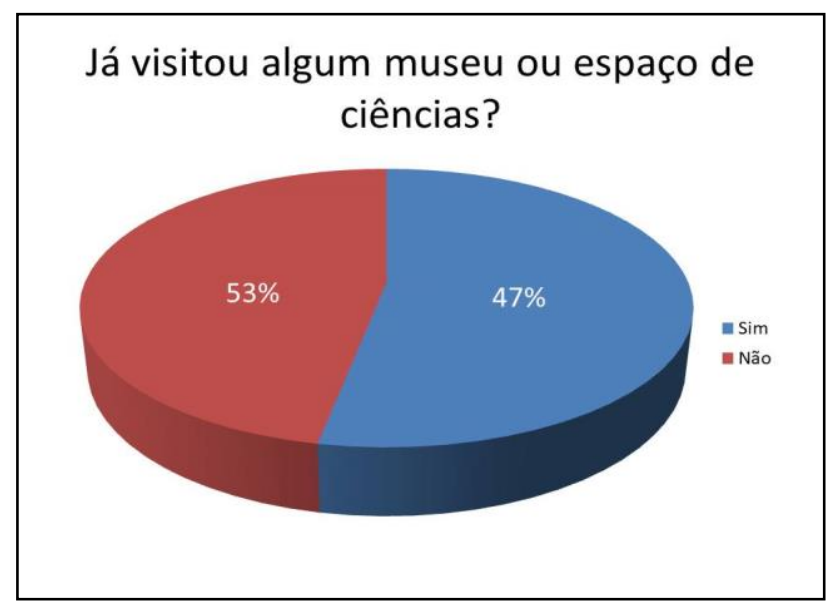

Figura 1. Já visitou algum museu ou espaço de ciências?

Esse dado corrobora com pesquisas realizadas ao longo da última década que apontam para o fato de grande parte da população brasileira não frequentar esse tipo de espaço. Segundo o Sistema de Indicadores de Percepção Social (SIPS), realizado pelo Instituto de Pesquisa Econômica Aplicada (IPEA), aproximadamente 68\% da população brasileira nunca realiza visitas a museus e centros culturais (SCHIAVINATTO, 2011). Essa realidade é ainda mais preocupante quando se considera a desigualdade social brasileira, permitindo identificar um fosso gigantesco no acesso a esses ambientes na comparação entre as classes mais elitizadas e as classes populares. 
Outro dado relevante é que dentre os estudantes que alegaram já ter ido a museus ou centros de ciências, a maioria disse que essas visitas foram acompanhadas pela escola (72\%) ou pela família (20\%) (Figura 2). Este fato evidencia a importância de iniciativas que, como o projeto "Descobrindo a Biofísica", promovem uma maior interação entre museus e escolas. Esta perspectiva coincide com muitos trabalhos que apontam para essa mesma conclusão (MARANDINO, 2001; KÖPTCKE, 2014).

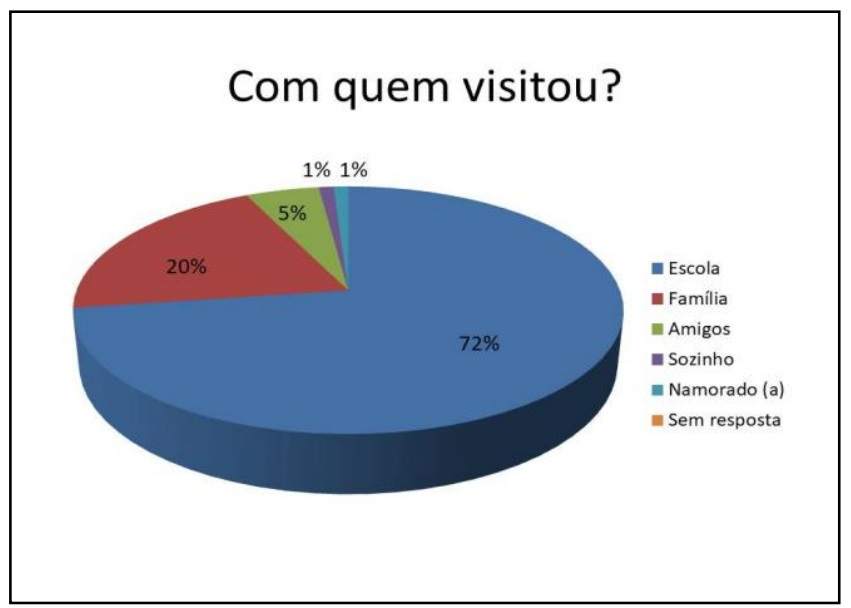

Figura 2. Caso tenha visitado, com quem visitou?

Os discentes que disseram nunca terem ido a um museu ou espaço de ciência atribuíram este fato principalmente à falta de oportunidades. Essa falta de oportunidade foi em grande parte generalizada, mas algumas respostas esclareceram melhor sua natureza, como por exemplo, falta de tempo (por parte do aluno ou dos pais) e a distância. O desinteresse em ir a esses espaços também apareceu, mas com uma frequência muito baixa (Figura 3).

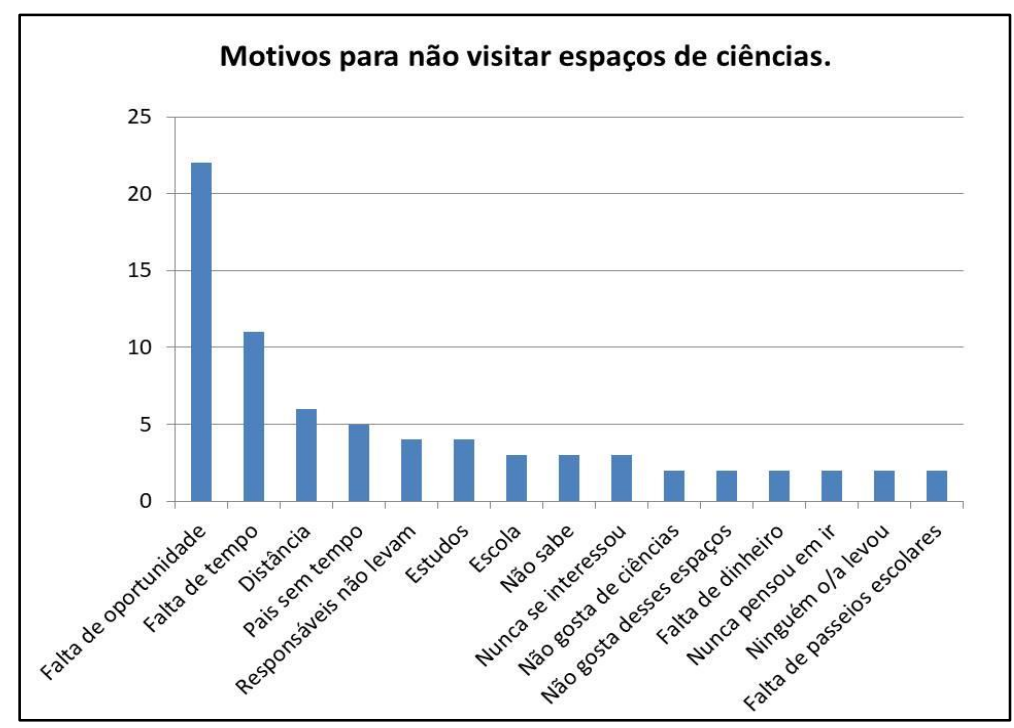

Figura 3. Motivos para não visitar espaços de ciências.

Em relação às respostas das perguntas abertas, destaca-se que aproximadamente $75 \%$ dos alunos classificam o objeto da exposição como sendo: Áreas da Ciência, Carlos Chagas Filho, Ciência e História. As respostas estão de acordo com os reais objetos da 
exposição, a não ser pelas respostas que a associam à figura de Carlos Chagas Filho (confusão compreensível em vista do fato de o início da visita acontecer no escritório do cientista). Os alunos indicam em suas considerações que as intervenções práticas tornam as visitas mais interessantes. Em análise paralela é possível traçar uma relação entre o interesse na exposição de ciências (com atividades práticas) e o desinteresse nas aulas de ciências (meramente expositivas), reflexo da falta de infraestrutura de muitas das escolas visitantes. A maioria dos alunos afirmou que voltaria ao EMCCF ou a outro museu, o que confirma que em algum nível que a experiência foi marcante e agradável.

Essa metodologia de análise encontra alguns limites. Em primeiro lugar, muitos alunos deixam respostas em branco, como o caso da pergunta sobre o motivo de não frequentar museus. Em segundo lugar, foi observado que alguns alunos copiavam as respostas uns dos outros. Uma das razões pode ser o medo de haver respostas "corretas" e respostas "incorretas", possível reflexo da forma como eles se defrontam com esse tipo de avaliação no cotidiano escolar. Tendo isso em vista, sempre antes de aplicar o questionário os mediadores explicavam que não existe um padrão não aceitável de resposta e os incentivam a responder com suas próprias palavras.

Tendo esses limites em mente, os próximos passos consistem em aumentar o quantitativo de questionários, visando ampliar nossos dados estatísticos e realizar grupos focais nas escolas que visitaram o museu e a exposição. Este tipo de técnica permite uma maior troca entre os participantes, o que pode permitir um conjunto maior de nuances. Geralmente, os próprios participantes levantam questões que não haviam sido inicialmente previstas pelos pesquisadores, enriquecendo, portanto, o estudo. Sendo assim, os grupos focais são de grande valor para examinar como as diferentes percepções se relacionam com o contexto sociocultural.

\section{Conclusões}

Ao longo dos últimos anos maiores esforços vêm sendo realizados para promover uma melhor compreensão sobre as possíveis contribuições de museus à sociedade. Nesse intervalo de tempo tem-se tornado claro que esses ambientes são capazes de produzir experiências prenhes de significados aos campos cultural, educacional e afetivo. Diante dessa perspectiva, o EMCCF desenvolveu mecanismos visando identificar e analisar a percepção de estudantes da educação básica sobre as experiências por eles vividas em suas visitas ao espaço. Ao longo deste trabalho foram apresentados alguns dos resultados obtidos nesse processo.

Uma importante característica sociocultural identificada por esse levantamento se relaciona com o fato de a maioria dos estudantes não costumarem visitar museus e centros de ciências, realidade justificada pela falta de oportunidades. Dentro desse mesmo item pode-se destacar que, segundo os estudantes que visitam com alguma frequência esse tipo de espaço, o incentivo das escolas é algo fundamental, o que aponta novamente para a importância de promover uma maior aproximação entre ambientes de educação formal e de educação não formal. 
Outro ponto relevante refere-se ao fato de os estudantes atribuírem grande destaque às atividades práticas realizadas ao longo da visita, as quais permitem uma maior interação do público com a exposição e com os temas por ela apresentados. Isso é bastante interessante, pois reforça em grande medida a necessidade de se realizar maiores investimentos em uma prática comunicativa inclusiva, que insira o público de maneira real no processo de mediação. É possível, ainda, indicar que essa maior inclusão possa ser feita nos museus, mas também nas instituições formais de ensino, locais que, via de regra, dão início à formação desses sujeitos e lhes apresentam grande parte das questões científicas mais elementares.

Por fim, após a análise dos dados obtidos foi possível concluir que é necessário dar continuidade ao Projeto "Descobrindo a Biofísica". Este projeto permite que estudantes de classes sociais menos privilegiadas conheçam o ambiente acadêmico e possam extrair dessa experiência momentos marcantes. Isto, em um cenário ideal, pode permitir que esses jovens percebam esse espaço como algo menos distante de suas realidades, já que se busca nessa visitas destacar que ocupar a universidade pública deve ser uma opção para eles também. Não se trata de realizar proselitismo, mas de permitir que estes possam ampliar suas possibilidades, reduzindo gradativamente o elitismo ainda existente no ambiente acadêmico, reflexo da histórica desigualdade socioeconômica e racial que atravanca nosso real desenvolvimento.

\section{Referências}

BONATTO, M. P. O.; SEIBEL, M. I; MENDES, I. A. Ação mediada em museus de Ciências: o caso do Museu da Vida. In: MASSARANI, L. et al. (org.). Diálogos \& Ciência: mediação em museus e centros de Ciência. Rio de Janeiro: Casa de Oswaldo Cruz/Fiocruz, 2007, p. 48-55.

CASTELFRANCHI, Y. O museu como catalisador de cidadania científica. Entrevista concedida a LOPES, S.C. In: MASSARANI, L., NEVES, R., AMORIN, L. (org.). Divulgação científica e museus de ciência: o olhar do visitante - Memórias do evento. Rio de Janeiro: Museu da Vida/Casa de Oswaldo Cruz/Fiocruz; RedPop, 2016, p. $37-46$.

KÖPTCKE, L. S.. Revisitando a parceria museu-escola: currículo e formação profissional. Museologia e Patrimônio, v. 7, p. 15-35, 2014.

MARANDINO, M.. Interfaces na Relação Museu-Escola. Caderno Catarinense de Ensino de Física, Florianópolis, v. 8, n.1, p. 85-100, 2001.

SCHIAVINATTO, F. Sistema de indicadores de percepção social (SIPS). 1.ed. Brasília: IPEA, 2011. 